\title{
Behavior of Power System Equilibrium Points in Dynamic Available Transfer Capability Calculation
}

\author{
Mohamed Shaaban * \\ Department of Electrical and Electronic Engineering, Faculty of Engineering, Universiti \\ Malaysia Sarawak, 94300 Kota Samarahan, Sarawak, Malaysia
}

\begin{abstract}
Available Transfer Capability (ATC) is of fundamental importance in electric power system planning and operation. The calculation of firm ATC in a power market environment is carried out based on day-ahead market dispatch with a set of security constraints. Incorporating dynamic security constraints into the ATC calculation not only renders a heavier burden on the computational approach, but also manifests complex system behavior in the neighborhood of its equilibrium points. This paper specifically highlights exotic system characteristics encountered during dynamic ATC calculation. The problem of ATC calculation is modeled as a nonlinear mathematical programming problem to maximize the power transfer subject to system technical and operating constraints. The dynamic ATC constraints are represented via the quadratic approximation of the stable manifold of the controlling unstable equilibrium point (UEP). A case study on the IEEE WECC 3-machine, 9-bus power system is presented and analyzed.
\end{abstract}

Keywords: Available transfer capability (ATC), electric power system, equilibrium point, optimization.

\section{Introduction}

Available Transfer Capability (ATC) is a crucial index in the competitive marketplace to quantify the unutilized extent of the transmission system to facilitate additional energy trading [1], [2]. ATC determination should include adequate uncertainties existing in the physical system structure such as load fluctuations and equipment availabilities. The latter can be accounted for by analyzing a credible set of system contingencies [3]. Independent systems operators (ISOs) calculate ATC on hourly basis and post its values online to reflect market conditions as the market timeline progresses from day-ahead through real-time [4]. ATC can also be calculated two days to few months in advance of the dispatch day, as with the current practices of the New York ISO [5].

Various approaches addressed the issue of ATC computations based on static security considerations [6]-[10]. Nonetheless, the task of calculating the ATC subject to dynamic security constraints remains far more challenging with fewer approaches available [11]. This is due to the fact that stressed power systems exhibit complex dynamic behaviour [12]. Furthermore, dynamic ATC calculation algorithms are based on mature technologies commonly used in transient stability studies. The environment at which the ATC is required to be calculated is different from traditional stability studies. This new environment is typically characterized by multiple energy transactions, market schedules and processes, as well as increased uncertainties due to the proliferation of variable

* Corresponding author. Tel.: +6-082-583410; fax: +6-082-583410

E-mail address: mshaaban@unimas.my

Manuscript History:

Received 16 January, 2018, Revised 5 March, 2018, Accepted 14 March, 2018, Published 31 March, 2018

e-ISSN: 2289-7771 
renewable generation connected to the grid. A calculation tool that identifies such a changing environment is therefore necessary.

In this paper, ATC is formulated as an optimization problem. Dynamic security constraints are included in the constraint set as a nonlinear equality representing the geometric quadratic approximation of the stable manifold of the controlling unstable equilibrium point (UEP). A case study, using a standard test system, is presented to show the unusual behavior of the equilibrium points of the system while being gradually overloaded.

\section{Formulation of the ATC problem}

A prospective power transfer between two given nodes (generator and load) in a power system can be cast as an optimization problem to maximize the function $f$ representing the sum of active power generated from some specific generator(s) with respect to some $\operatorname{load}(\mathrm{s})$, as constrained by network and equipment limits, in addition to a number of credible outage contingencies. This can be constructed generally as:

$$
\underset{u_{0}}{\operatorname{Maximize}} f\left(x_{0}, u_{0}\right)
$$

Subject to:

$$
\begin{aligned}
& g_{0}\left(x_{0}, u_{0}\right)=0 \\
& h_{0}\left(x_{0}, u_{0}\right) \leq 0 \\
& g_{p}\left(x_{0}, z_{p}, u_{0}\right)=0 \\
& h_{p}\left(x_{p}, z_{p}, u_{0}\right) \leq 0 \\
& \dot{z}_{p}=\Gamma\left(z_{p}(t), x_{p}(t), u_{0}\right)=0 \\
& p=1,2,3, \ldots, n_{c}
\end{aligned}
$$

where $u_{0}$ is the control variable and $x$ is the algebraic state variables comprising bus voltage magnitudes and angles. $g_{0}$ is the power flow equality constraints and $h_{0}$ is the technical and operational system and equipment limits. $g_{p}$ and $h_{p}$ are the equality and inequality constraints for the contingency $p . \Gamma$ is a function representing power system dynamics, whereas $z_{p}$ denotes the dynamic state variables such as machine angles and velocities corresponding to contingency $p . n_{c}$ is the number of contingencies.

The formulation in (1) is a nonlinear optimization problem with differential and algebraic equations (DAE) as constraints. Conventional optimization approaches cannot directly handle differential equations. On the other hand, discretizing the differential equations into a set of algebraic equations introduces a very large number of constraints into the formulation that could well lead to convergence problems. 


\section{Solution approach}

The intricacy of the formulation in (1) coupled with the current trend of decentralization and incomplete system information, since generators are owned and operated by independent power producers (IPPs) or private generation companies (Gencos), suggest the use of decomposition techniques. In this paper, Benders decomposition is used to separate (1) into a master problem containing the non-contingency base case (1.a and 1.b) along with sub-problems representing contingency cases (1.c-1.e). Detailed application of the Benders decomposition to separate the problem (1) into a master problem and a slave subproblem was thoroughly reported in [9].

Transient stability limit determination is carried out using direct methods which provide a quantitative estimate of the stability region of the power system [13]. The stability criterion in this case is to study whether the postfault system trajectory would converge to the postfault stable equilibrium point (SEP) as time proceeds. Under certain nondegeneracy conditions, the stability boundary of the nonlinear power system described in (1.e) is the union of the stable manifolds of the unstable equilibrium points (UEPs) lying on the boundary [13], [14]. The controlling UEP is the UEP whose stable manifold contains the point where the faulted system trajectory exits the stability boundary of the postfault system [14]. Since an exact computation of the stable manifold of the relevant UEP is quite involved, the stable manifold of the UEP can be typically approximated with some local validity [15], [16].

In this paper, the computation of the controlling UEP is carried out using the BCU method [13], whereas the stability boundary is determined via the numerical quadratic approximation of the stable manifold anchoring the controlling UEP [16]. The resulting expression is integrated with the optimization framework (1).

A flowchart of the solution approach is outlined in Figure 1. Voltage magnitudes and angles are first initialized to a flat start. Contingency $p$ is then identified and the iteration counter $k$ is reset as well. The master problem is then solved and the control variable $u_{0}$ is determined (a set of active power injections and extractions). The SEP and the controlling UEP corresponding to the postfault system of contingency $p$ are then calculated. The dynamic security constraint is then computed, at the controlling UEP, and the slave subproblem is solved. If there is any limit violation, the iteration counter is advanced and the master problem is solved again to obtain a new set of MW injections that satisfy the constraints. The process is then repeated until there is no limit violation. If no violations occur, another contingency is taken into consideration and the approach proceeds to solve the subproblem corresponding to the new contingency case. The whole procedure is repeated all-over again until all specified contingencies are contemplated.

\section{Case study}

The proposed approach is applied to the WECC 3-machine, 9-bus power system, a standard IEEE test system, as shown in Fig. 2. The parameters of the power system and the operating point are listed in [12]. Using relative machine formulation, machine $n$ was taken as a reference node assuming it as an infinite bus with very large inertia [13]. In this case, machine 3 is taken as a reference with its rotor angle $\delta_{3}=0$. The classical model of a generator, represented by a constant voltage behind a transient reactance, is adopted [12]. Loads are modeled as constant impedances and are eliminated by the network reduction model. Generators have a uniform damping of 0.1 .

Given a $250 \mathrm{MW}$-multilateral transaction to take place between buses 1 and 2 as seller generators with buses 5 and 6 as buyers, ATC needs to be assessed to check the physical viability of this transaction. Analysis of the base-case (non-contingency) condition shows that, ATC value is 200 MW. Benders decomposition is invoked to assess the impact of system contingencies [9]. Considering single line contingencies and steady-state constraints only, ATC is found to be $36 \mathrm{MW}$ due to the outage of line 5-7. It is assumed that a three phase to ground fault takes place at line 5-7 and very 
close to bus 7; with the fault cleared at 0.1s. The quadratic approximation of the stable manifold is computed [16] and incorporated in (1). ATC is $31.5 \mathrm{MW}$ due to the dynamic constraints corresponding to the outage of line 5-7. It is quite clear how the system dynamics could be the binding constraint for physical MW power transactions across the grid. In this case, consideration of the contingency, from a static point of view, has reduced the ATC value to be $18 \%$ of its corresponding non-contingency case. Moreover, due to the dynamic system constraints, ATC is further reduced to almost $15 \%$ of the non-contingency ATC value.

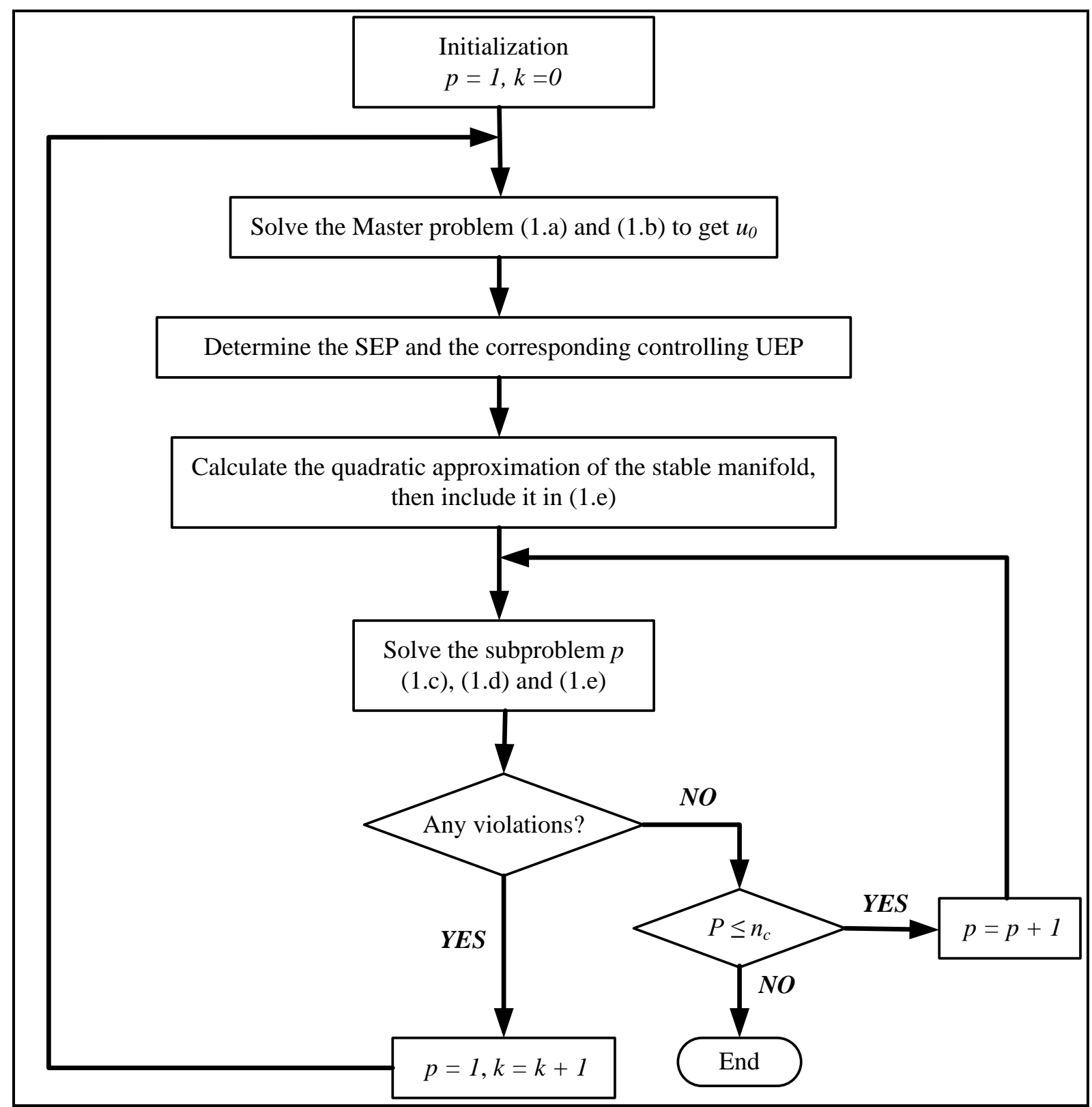

Figure 1. Flowchart of the solution approach.

In the due process of calculating the dynamic ATC, the SEPs and the corresponding controlling UEPs are computed as illustrated Fig. 3. Relaxing some of the constraints and further calculating the ATC and the respective SEPs and UEPs indicate that higher the ATC value, the closer the SEPs and UEPs move towards each other in a linear pattern. When the ATC is $95 \mathrm{MW}$, the SEP and UEP 
coalesces and the SEP disappears altogether [15], [17]. It is plausible that the increase in the ATC estimates, associated with additional transactions, causes shrinkage in the stability region of the system. This, in turn, raises the possibility that the system becomes unstable. At a specific value of the ATC (i.e., $95 \mathrm{MW}$ ), the stability region completely vanishes. In this case, the proposed $250 \mathrm{MW}$ multilateral transaction between selling and buying buses can never be approved by the ISO under given system conditions.

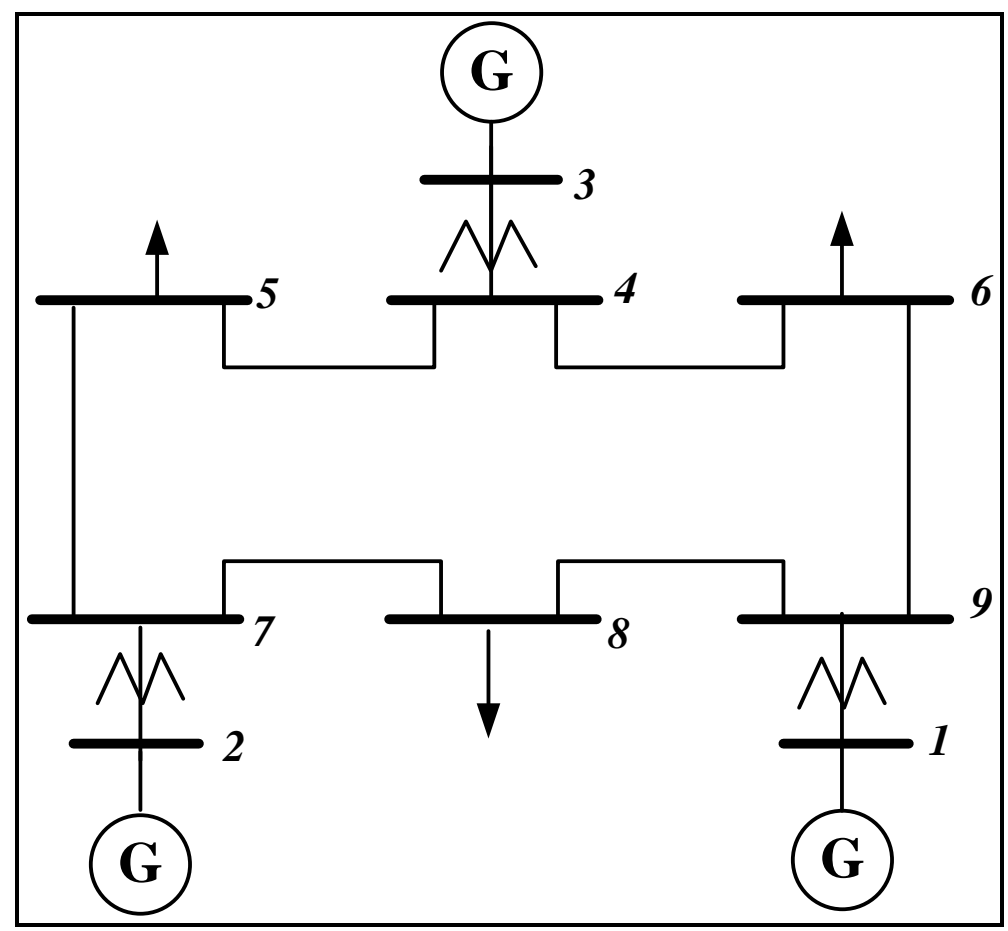

Figure 2. WECC 3-generator, 9-bus system.

This behavior of the equilibrium points (corresponding to ATC value of $95 \mathrm{MW}$ ), nonetheless, corresponds to a saddle-node bifurcation or the maximum system loading. This kind of bifurcation is usually associated with voltage collapse in electric power systems. Voltage collapse is a form of instability of heavily loaded power systems which leads to declining voltages and ultimately a blackout [18]. It appears that the loading direction in this case does not coincide with the critical direction in the load space. This could be attributed to the fact that in this calculation, we were interested in the relevant or controlling UEP of the faulted system trajectory.

On the other hand, the path of the stable and unstable equilibria movement is shown to be almost linear, given that there is a particular direction of load increase and specific generator participation factors as stipulated by the proposed transaction. The preceding property may be used to estimate the controlling UEP at different ATC values from the SEP of the postfault initial operating condition and the corresponding SEPs of incremental ATC estimates. Since the computation of the controlling UEP using the BCU method requires numerical integration of system dynamic equations, this property can substantially minimize the computational burden associated with dynamic ATC computations. 


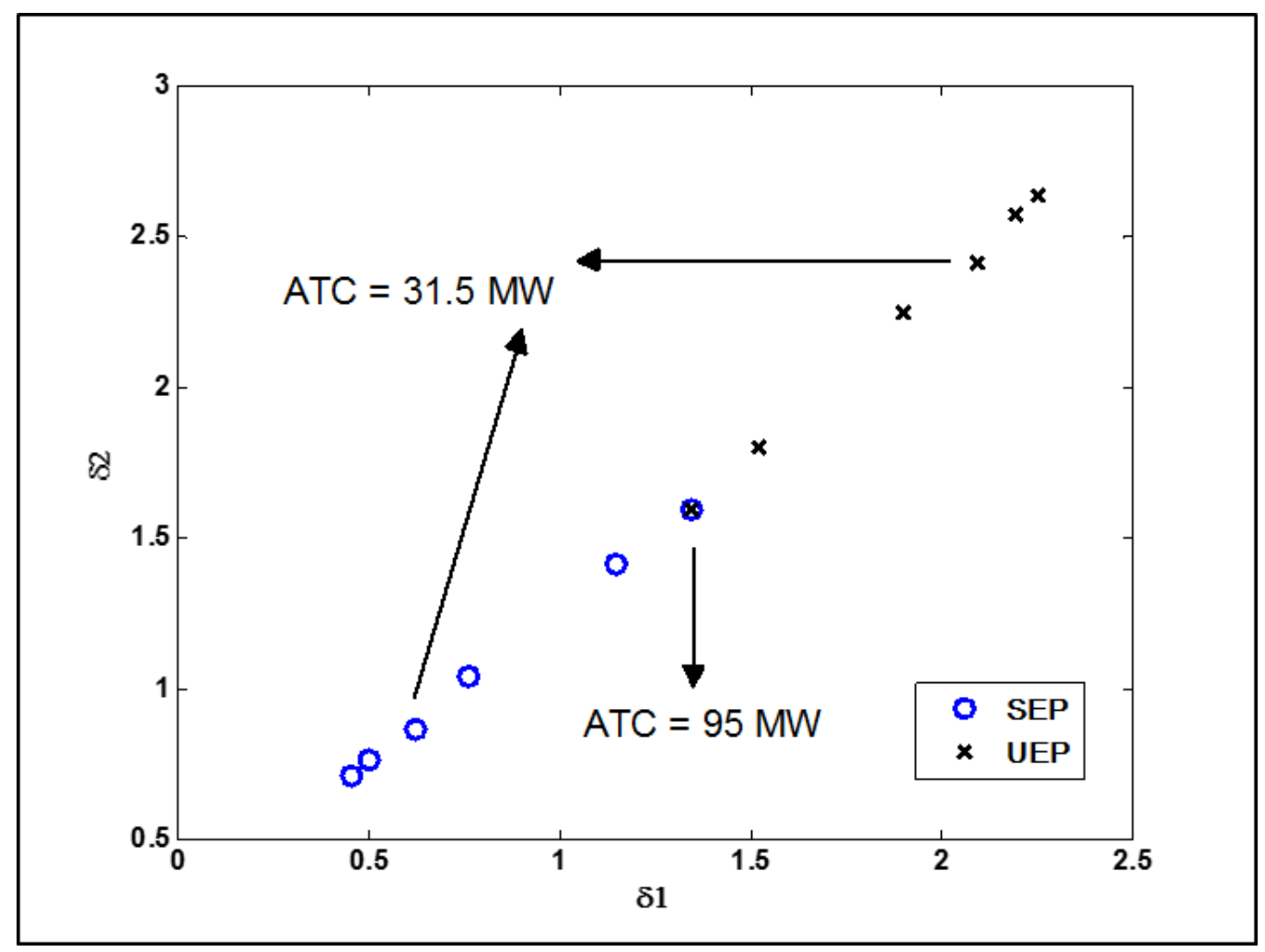

Figure 3. SEPs and UEPs a for the outage of line 5-7 (angles in radians).

\section{Conclusion}

Due to the trend of liberalization in power generation and the increase in MW transactions across the system, unprecedented operation scenarios are emerging. In these scenarios, the electric power system can be driven into stressed conditions, operating closer to its physical limits. The paper has briefly described a method to calculate the ATC of a power system considering dynamic security constraints. It has provided an additional insight into the peculiar characteristics of the power system near its equilibrium points during dynamic ATC calculations. It was shown that the SEP and the controlling UEP collide, with the SEP annihilated, resulting in the disappearance of the stability region altogether.

The behavior of the power system equilibrium points under various loading levels is still ambiguous. Further studies are needed to better understand the global dynamics of power systems with bulk power transactions and complex operation paradigms.

\section{Acknowledgements}

This research was supported by Universiti Malaysia Sarawak (UNIMAS) via Special Grant Scheme (SpGS), Grant No. F02/SpGS/1544/2017. 


\section{References}

[1] Wei, Z., Chen, S., Sun, G., Wang, D., Sun, Y. and Zang, H. (2016). Probabilistic Available Transfer Capability Calculation Considering Static Security Constraints and Uncertainties of Electricity-Gas Integrated Energy Systems. Applied Energy, Vol. 167, 305-316.

[2] Mushfiq-Ur-Rahman, K., Saiduzzaman, M., Mahmood, M. N. and Khan, M. R. (2013). Calculation of Available Transfer Capability (ATC) of Bangladesh Power System Network. IEEE Innovative Smart Grid Technologies-Asia (ISGT-Asia), Bangalore, India, 1-5.

[3] Transmission Transfer Capability Task Force (1996). Available Transfer Capability Definitions and Determination, North American Electric Reliability Council (NERC), Princeton, NJ, USA.

[4] Price, J. E. (2011). Management of Available Transfer Capability with Growth in Renewable Resources. IEEE Power and Energy Society General Meeting. Detroit, MI, USA, 1-8.

[5] ISO New York. Http://mis.nyiso.com/public/P-8list.htm. [Last Access]: 03/03/2018.

[6] Ejebe, G. C., Waight, J. G., Santos-Nieto, M. and Tinney, W. F. (2000). Fast Calculation of Linear Available Transfer Capability. IEEE Transactions on Power Systems. Vol. 15, No. 3, 1112-1116.

[7] Khosravifard, M. and Shaaban, M. (2015). Risk-Based Available Transfer Capability Assessment Including Nondispatchable Wind Generation. International Transactions on Electrical Energy Systems. Vol. 25, No. 11, 3169-3183.

[8] Pandey, S. N., Pandey, N. K., Tapaswi, S. and Srivastava, L. (2010). Neural Network-Based Approach for ATC Estimation Using Distributed Computing. IEEE Transactions on Power Systems. Vol. 25, No. 3, 1291-1300.

[9] Shaaban, M., Li, W., Liu, H., Yan, Z., Ni, Y. and Wu, F. (2003). ATC Calculation with Steady-State Security Constraints Using Benders Decomposition. IEE Proceedings- Generation, Transmission and Distribution. Vol. 150, No. 5, 611-615.

[10] Shaaban, M., Li, W., Yan, Z., Ni, Y. X. and Wu, F. F. (2003). Calculation of Total Transfer Capability Incorporating the Effect of Reactive Power. Electric Power Systems Research. Vol. 64, No. 3, 181-188.

[11] Jain, T., Singh, S. N. and Srivastava, S. C. (2008). Dynamic Available Transfer Capability Computation Using a Hybrid Approach. IET Generation, Transmission \& Distribution. Vol. 2, No. 6, 775-788.

[12] Anderson, P. M. and Fouad, A. A. (2008). Power System Control and Stability, 2nd ed., Wiley India PVT Limited.

[13] Chiang, H. D., Wu, F. F. and Varaiya, P. P. (1994). A BCU Method for Direct Analysis of Power System Transient Stability. IEEE Transactions on Power Systems. Vol. 9, No. 3, 1194-1208.

[14] Alberto, L. F. C. and Chiang, H. D. (2012). Characterization of Stability Region for General Autonomous Nonlinear Dynamical Systems. IEEE Transactions on Automatic Control. Vol. 57, No. 6, 1564-1569.

[15] Saha, S., Fouad, A. A., Kliemann, W. H. and Vittal, V. (1997). Stability Boundary Approximation of a Power System Using the Real Normal Form of Vector Fields. IEEE Transactions on Power Systems. Vol. 12, No. 2, 797-802.

[16] Venkatasubramanian, V. and Ji, W. (1997). Numerical Approximation of (n-1)-Dimensional Stable Manifolds in Large Systems such as the Power System. Automatica. Vol. 33, No. 10, 1877-1883.

[17] Berggren, B. and Andersson, G. (1993). On the Nature of Unstable Equilibrium Points in Power Systems. IEEE Transactions on Power Systems. Vol. 8, No. 2, 738-745.

[18] Dobson, I. (1992). Observation on the Geometry of Saddle Node Bifurcation and Voltage Collapse in Electrical Power Systems. IEEE Transactions on Circuits and Systems-I: Fundamental Theory and Applications. Vol. 39, No. 3, 240-243. 